\title{
Synchronization of active mechanical oscillators by an inertial load
}

\author{
Andrej Vilfan ${ }^{1,2, *}$ and Thomas Duke ${ }^{1, \dagger}$ \\ ${ }^{1}$ Cavendish Laboratory, Madingley Road, Cambridge CB3 OHE, UK \\ ${ }^{2} J$. Stefan Instutute, Jamova 39, 1000 Ljubljana, Slovenia
}

(Dated: February 2, 2008)

\begin{abstract}
Motivated by the operation of myogenic (self-oscillatory) insect flight muscle, we study a model consisting of a large number of identical oscillatory contractile elements joined in a chain, whose end is attached to a damped mass-spring oscillator. When the inertial load is small, the serial coupling favors an antisynchronous state in which the extension of one oscillator is compensated by the contraction of another, in order to preserve the total length. However, a sufficiently massive load can sychronize the oscillators and can even induce oscillation in situations where isolated elements would be stable. The system has a complex phase diagram displaying quiescent, synchronous and antisynchrononous phases, as well as an unsual asynchronous phase in which the total length of the chain oscillates at a different frequency from the individual active elements.
\end{abstract}

PACS numbers: $05.45 . \mathrm{Xt}, 87.19 . \mathrm{Ff}, 87.16 . \mathrm{Nn}$

The origin of movement in biological systems can frequently be traced to molecular motors - specialized proteins that convert chemical energy to mechanical work. Kinesin and dynein, which travel along microtubules, and myosin, which pulls on actin filaments, are typical examples 1, 2]. In many cases, such as muscle contraction or intracellular transport, molecular motors generate unidirectional motion. But there are also a number of physiological systems which incorporate motor proteins that display oscillatory dynamics. These include eucaryotic flagella and cilia whose undulation is driven by dynein molecules, and the flight muscles of many insects which contract rythmically at a frequency that is out of step with the excitory neural impulses $3,[4,[5,6,67,8$. Apparently these systems are self-oscillatory, and the dynamical instability that leads to vibration is directly generated by the action of the motor proteins; it is usually attributed to delayed stretch activation, which can be caused by a variety of different microscopic mechanisms (reviewed in Refs. [6] \& [7]). Oscillations have also been observed in the sarcomeres of skeletal muscle in non-physiological conditions [9, 10, 11, 12] and in experiments that probe the interaction between individual dynein molecules and microtubules 13]. Even normal muscle fibers can display a damped oscillatory response to sudden changes in load 14].

Theoretical analysis has demonstrated that a single filament interacting with an ensemble of motors can have an anomalous force-velocity relation [15, 16], whereby two different sliding speeds, one positive and one negative, can occur at a given load. Experimental confirmation of this phenomenon has been obtained in gliding motility assays for both actin- 17] and microtubulebased systems [18]. In such a situation the motors can collectively generate oscillations when the filament is con-

*Andrej.Vilfan@ijs.si

†td18@cam.ac.uk

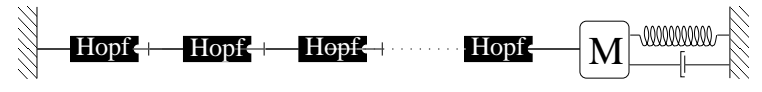

FIG. 1: Model system: A chain of serially coupled active Hopf oscillators is attached to a damped inertial oscillator.

nected in series with an elastic element 19, 20, 21], because the solution for the sliding speed then switches periodically between the two stable branches. However, direct application of this model to muscle fibers, composed of hundreds of sarcomeres (contractile units) in series, omits a crucial point: the oscillations will only be macroscopically observable if there is at least some degree of synchrony between the oscillations of individual sarcomeres. How might this occur? One possibility is that the activity of myosin motors in different sarcomeres is coordinated by some chemical signaling. An alternative suggestion is that torsion of actin filaments is involved 11]. In this Letter, we propose a mechanism of synchronization that does not rely on any such specific molecular process. We investigate the dynamics of a chain of active mechanical elements each of which, when isolated, can undergo a dynamical instability from a quiescent (stable) to an oscillatory (unstable) regime. In the case where each element is individually stable, we show that the entire chain can be set into synchronized vibration by the application of a sufficiently massive inertial load at its end. In the alternative case where each contractile element is unstable, we demonstrate the existence of a variety of dynamical regimes, including an unusual asynchronous state in which individual elements oscillate at a faster frequency than the mass to which they are connected.

The model system that we investigate is shown in Fig. 11 Each contractile element in the chain is an active mechanical system whose displacement $x_{i}$ is coupled nonlinearly to an internal variable $y_{i}$ (which might, for example, be the fraction of bound motors, or the concen- 
tration of a regulator). We suppose that an element may either stay still or generate self-sustained oscillations, depending on the value of a control parameter. In the vicinity of the critical point where the quiescent state becomes unstable, the dynamics may be described by the canonical equation for a Hopf bifurcation. Writing the complex variable $z_{i}=x_{i}+\mathrm{i} y_{i}$, we have

$$
\dot{z}_{i}=(\mathrm{i} \omega+\epsilon) z_{i}-B\left|z_{i}\right|^{2} z_{i}+F / \zeta,
$$

where $\epsilon$ is the control parameter and $\omega$ is the characteristic frequency of each active oscillator. $F$ is the force acting on each of the oscillators in the chain, due to the coupled mass-spring system and is determined by its equation of motion:

$$
F=-M \sum_{i=1}^{N} \operatorname{Re}\left(\ddot{z}_{i}+\Omega^{2} z_{i}+\gamma \dot{z}_{i}\right)
$$

where $M$ is the mass of the load, $\gamma$ is a measure of the damping and $\Omega$ is the natural frequency of the massspring system. In order to proceed further with the analysis and obtain numerical solutions, we replace Eq. (2) with a first-order differential equation by inserting $\ddot{z}_{i}$ from the first derivative of Eq. (11)

$$
\begin{aligned}
& \dot{F}=- \frac{\zeta}{N M} F+\frac{\zeta}{N} \sum_{i=1}^{N}\left(-\epsilon \operatorname{Re} \dot{z}_{i}+\omega \operatorname{Im} \dot{z}_{i}\right. \\
&\left.+B \operatorname{Re} \frac{d\left(z_{i}^{2} z_{i}^{*}\right)}{d t}-\Omega^{2} \operatorname{Re} z_{i}-\gamma \operatorname{Re} \dot{z}_{i}\right)
\end{aligned}
$$

where the terms containing $\dot{z}_{i}$ can be substituted from Eq. (11).

These equations constitute a system of globally coupled oscillators [22, 23, 24, 25] (the oscillators interact with each other via the single variable $F$ ). However, our model differs in a crucial way from classical models of synchronization, such as the Kuramoto model [26], in that the coupling variable $F$ is determined by a first-order differential equation (Eq. 3), rather than as a function of the variables $z_{i}$. By expressing all frequencies in terms of $\omega$ and amplitudes in terms of $\sqrt{\omega / B}$, and assuming $N \gg 1$, the number of model parameters is reduced to four: $\epsilon / \omega, \Omega / \omega, \gamma / \omega$ and $\zeta / N M \omega$.

We start our analysis by considering the situation in which the active elements would be stable if isolated, $\epsilon<0$. With $\epsilon$ fixed, the frequency of the inertial oscillator $\Omega$ acts as a control parameter for the system as a whole. We can identify a transition between a quiescent phase (for $\Omega<\Omega_{c}$ ) in which the entire system is at rest, and a synchronized phase (for $\Omega>\Omega_{c}$ ) in which all of the contractile elements and the massive load oscillate together (see Fig. 2). At the critical value $\Omega=\Omega_{c}$ the system undergoes a Hopf bifurcation. In its vicinity we can use linear stability analysis, because the amplitude tends to zero there. In the synchronized phase, all oscillators have identical displacement $z_{i} \equiv z=x+\mathrm{i} y$ and Eqs. (11) \& (3) form a system of three coupled differential
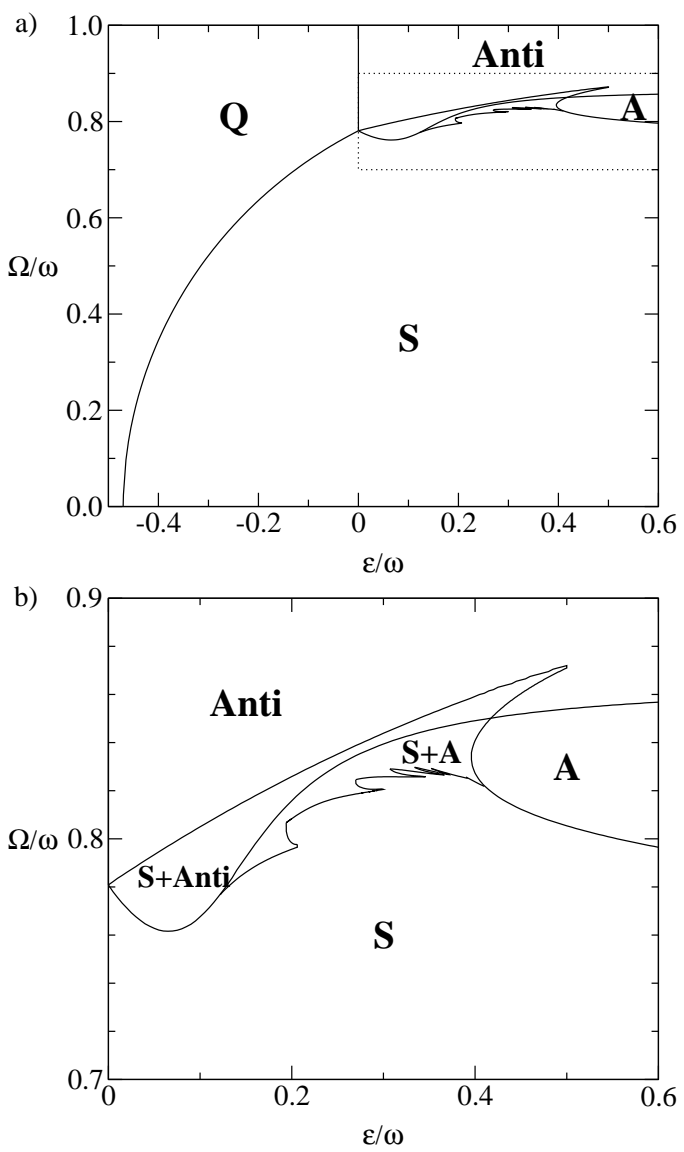

FIG. 2: Phase diagram (Q quiescent; S synchronous; Anti antisynchronous; A asynchronous) as a function of the control parameter $\epsilon$ of the active elements and the frequency $\Omega$ of the inertial oscillator. Other parameters have the values $\zeta / N M \omega=0.3125$ and $\gamma / \omega=0.2$. The diagram was established by numerical solution of Eqs. (1) \& (3). The phase boundary of the synchronous state is given by Eq. (4) for $\epsilon<0$ and can be determined by perturbation theory for $\epsilon>0$.

equations for $x, y$ and $F$. The characteristic equation for the eigenvalues of the Jacobian reads $\lambda^{3}-(\epsilon-\zeta / N M-$ $\gamma) \lambda^{2}+\left(\Omega^{2}-\epsilon \gamma-2 \epsilon \zeta / N M\right) \lambda-\Omega^{2} \epsilon+\left(\epsilon^{2}+\omega^{2}\right) \zeta / N M=0$. The transition occurs when the real part of the complex eigenvalue pair changes sign, giving

$$
\Omega_{c}=\sqrt{\omega^{2} /\left(1+\frac{N M \gamma}{\zeta}\right)+\epsilon\left(\gamma-\epsilon+2 \frac{\zeta}{N M}\right)} .
$$

The frequency $f$ of synchronized oscillations at the bifurcation is given by the imaginary part of the complex eigenvalues:

$$
f=\sqrt{\omega^{2} /(1+\gamma N M / \zeta)-\epsilon^{2}} .
$$

Note that it is always the case that $\Omega_{c}<f<\omega$. Away from the bifurcation, $\Omega<\Omega_{c}$, the synchronized oscillations have a frequency lower than $f$, thus $f$ represents the maximal frequency of the system. 

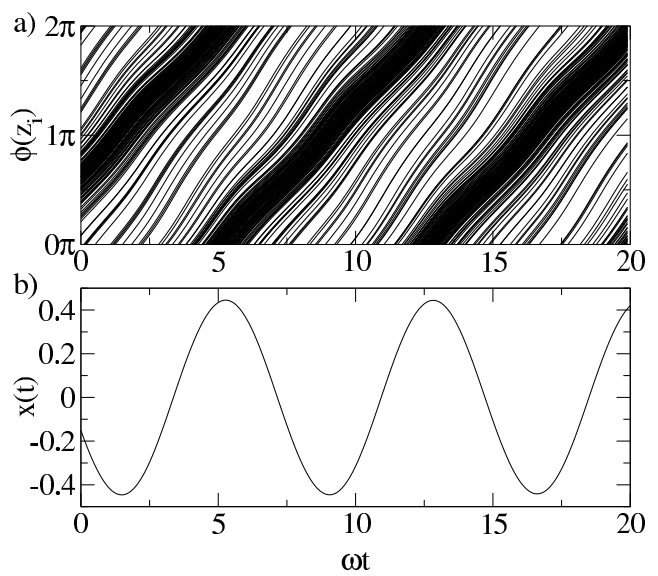

FIG. 3: The asynchronous state. (a) Phase $\phi_{i}=\arg \left(z_{i}\right)$ of each of the $N=128$ active elements as a function of time. The phase velocity $\dot{\phi}_{i}$ of a particular element is slow for a number of periods of oscillation, then speeds up over a few periods, then slows again. The average frequency of the active elements is therefore faster than the speed of propagation of the the high-density area in the phase space, which is the frequency of the inertial load. (b) Total extension $x=\Sigma_{i} \operatorname{Re} z_{i}$ (in units $N \sqrt{\omega / B}$ ) as a function of time, illustrating the lower frequency of the inertial oscillator. $\mathrm{Pa}$ rameters: $\zeta / N M \omega=0.3125, \gamma / \omega=0.2, \epsilon / \omega=0.6$ and $\Omega / \omega=0.83$.

To interpret this result in terms of the underlying physical model, we can consider two different ways of adjusting the load. In the first, illustrated in Fig. 2a, we fix the mass and the damping of the inertial oscillator (thus $M=$ const, $\gamma=$ const) and change the stiffness of the spring (a stiffer spring implies a higher frequency $\Omega$ and vice versa). Then a sufficiently strong spring, such that $\Omega>\Omega_{c}$, will always maintain the stability of the system. But decreasing the stiffness can provoke a transition to synchronized oscillations, provided that the control parameter $\epsilon$ lies above some threshold value, determined by the solution $\Omega_{c}=0$ of Eq. (44) (i.e. the active elements must be sufficiently close to their dynamical instability). We also note that the chain can oscillate synchronously even when the inertial oscillator itself is overdamped (i.e. $Q=\Omega / \gamma<1$ ). In the second situation, we vary the mass $M$ of the inertial oscillator while keeping the spring stiffness $M \Omega^{2}$ and the damping $M \gamma$ constant. Now we find that the system will remain quiescent if the mass is sufficiently small, but that a larger mass can make the system oscillate provided that $\epsilon>-\omega / \sqrt{1+N M \gamma / \zeta}$.

We continue the analysis by considering the situation where the active elements are individually unstable, $\epsilon>0$, and would oscillate spontaneously if isolated. In this case the system displays a greater variety of phases, as indicated in Fig. 2] For small values of $\Omega$, there is a synchronous phase as described above. For large values of $\Omega$ there is an antisynchronous phase: All of the active

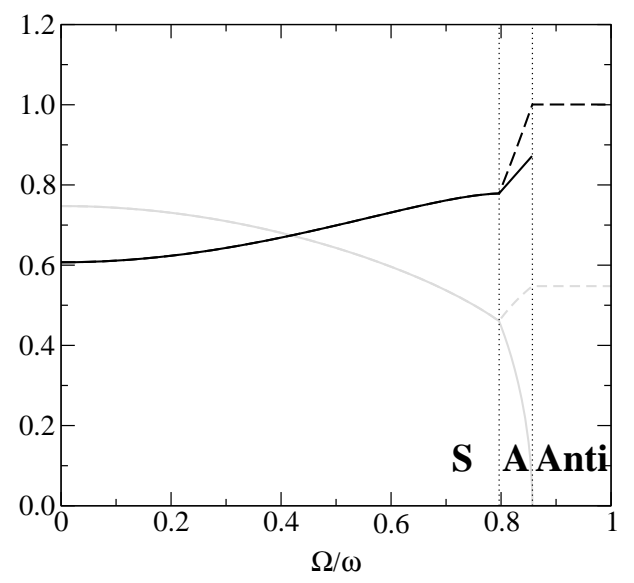

FIG. 4: Frequency and amplitude of oscillations as a function of $\Omega$ for a fixed, positive value of the control parameter $(\epsilon / \omega=0.6)$. These parameters correspond to moving up the right vertical axis of Fig. 2\%. The system transits from the synchronous (S), to the asynchronous (A), to the antisynchronous (Anti) phase. Black curves show the frequencies of the inertial load (continuous) and of the active elements (dashed). Gray curves show the RMS amplitude of the mass (continuous) in units $N \sqrt{\omega / B}$ and that of each active element (dashed) in units $\sqrt{\omega / B}$. Other parameters: $\zeta / N M \omega=0.3125, \gamma / \omega=0.2$.

elements oscillate with the same frequency $\omega$, but with a distribution of phases $\phi_{i}=\arg \left(z_{i}\right)$ such that the sum of their extensions is always zero; thus the massive load remains stationary. When the inertial frequency $\Omega$ quite closely matches the characteristic frequency $\omega$ of the active oscillators, there is a remarkable phase in which the individual elements oscillate at one frequency, while the total extension oscillates at a different, lower frequency. We label this phase asynchronous. As shown in Fig. 3 active oscillators form clusters with different phases $\phi_{i}$ and their phase velocities $\dot{\phi}_{i}$ periodically slow down and speed up. The number of oscillators in each cluster generally decreases with increasing $\epsilon / \omega$ and can be as small as one, which is the case in the example shown in Fig. 3 The ensemble of oscillators resembles a set of vehicles on a congested ring road, which repeatedly enter a traffic jam. Because vehicles enter the jam at its rear end and leave at the front, the jam progresses more slowly than the average speed of an individual vehicle. In our model, the total extension depends on the phase of the majority of active oscillators, and the oscillation frequency of the inertial load therefore corresponds to the speed of propagation of the traffic jam.

The dependencies on $\Omega$ of the frequencies of individual elements, and of the total displacement, are shown in Fig. [4 Also shown are the corresponding amplitudes of motion. In the synchronous phase, both frequencies are equal and the amplitude of the inertial load is $N$ times greater than that of each active element. In the asynchronous phase, the amplitude of the inertial oscil- 
lator falls towards zero, while that of the active elements approaches $\sqrt{\epsilon / B}$ - the amplitude of spontaneous oscillations for an isolated element. At the same time, the frequency of the active oscillators approaches their characteristic frequency $\omega$. In the antisynchronous phase the frequency and amplitude of the active elements remain unchanging at these limiting values, and the inertial mass does not move. For large values of $\epsilon$ all transitions are second order, and the system passes directly from the synchronized, to the asynchronous, to the antisychronous phase as $\Omega$ increases. For small, positive values of $\epsilon$ the transitions become first order and there is a region in which the synchronous phase and the asynchronous phase coexist as two metastable states. At intermediate values of $\epsilon$, a complex asynchronous phase arises in which three or more clusters of oscillators are synchronized within each cluster, but the clusters are out of phase with each other (see Fig. 20).

One experimental situation which appears to correspond to the regime $\epsilon>0$ is the spontaneous oscillatory contraction (SPOC) of skeletal muscle in conditions of high ADP/ATP ratio. Oscillations of the length of a myofilament have been observed when its end was attached to a flexible microneedle [11], and oscillations in the length of individual sarcomeres have been seen when the total length was held fixed [9]. These correspond to the synchronous and antisynchronous phases of our model. Additionally a 'metachronal' phase, in which contractile waves propagate along a myofilament, has been observed [10]. This cannot be explained by our model. It would require either chemical signaling between adjacent sarcomeres, or a gradient in one or more of the sarcomere properties (e.g. the number of myosin molecules interacting with the thin filament) as suggested by Smith and Stephenson [27.

The mechanics of insect flight muscle has been investigated in detail by Machin and Pringle [4, 5]. They found that a sudden increase in fiber length caused a subsequent rise in tension - the phenomenon known as 'delayed stretch activation'. Consequently a muscle subjected to a sinusoidal change of length produced net work [5]. They also observed that a muscle could be made to oscillate by attaching it to an inertial load [4], provided that the damping was not too great. They suggested that the load must be resonant (i.e. $Q=\Omega / \gamma>1$ ) for oscillations to occur, and found that the frequency of vibration was primarily determined by the inertia and elasticity of the load. All of these results are consistent with our model in the regime $\epsilon<0$ and $\zeta / N M \omega \ll 1$. But we predict that it is not in general necessary for the load to be resonant; oscillations should be observable whenever $\Omega$ is smaller than $\Omega_{c}$, given by Eq. (4). And we note that the oscillation frequency generally depends on the characteristic frequency $\omega$ of the sarcomeres, as well as on the nature of the inertial load. It is often stated that the wings and thorax of insects provide a resonant load whose oscillation is maintained by energy supplied by the flight muscles $[7,8]$. In the light of our investigation, it would be interesting to conduct experiments in which the mass and damping of this load is modified, to verify whether the muscles can generate oscillations in the absence of resonance.

\section{Acknowledgments}

We have benefited from discussions with Martin Falcke and thank Karsten Kruse for a critical reading of the manuscript. A.V. would like to acknowledge support from the European Union through a Marie Curie Fellowship (No. HPMFCT-2000-00522) and from the Slovenian Office of Science (Grant No. Z1-4509-0106-02).
[1] B. Alberts, D. Bray, J. Lewis, M. Raff, K. Roberts, and J. D. Watson, Molecular Biology of the cell (Garland Publ., New York, 1994), $3^{\text {rd }}$ ed.

[2] J. Howard, Mechanics of Motor Proteins and the Cytoskeleton (Sinauer, Sunderland, MA, 2001).

[3] These flight muscles are variously called 'myogenic', 'fibrillar', or 'asynchronous'; this last term refers to the fact that contraction is not directly related to the nerve impulses and has nothing to do with the synchronization of sarcomere oscillations discussed in this Letter.

[4] K. E. Machin and J. W. S. Pringle, Proc. R. Soc. Lond. B 151, 204 (1959).

[5] K. E. Machin and J. W. S. Pringle, Proc. R. Soc. Lond. B 152, 311 (1960).

[6] J. W. S. Pringle, Proc. R. Soc. Lond. B 201, 107 (1978).

[7] M. H. Dickinson and M. S. Tu, Comp. Biochem. Physiol. 116A, 223 (1997).

[8] R. K. Josephson, J. G. Malamud, and D. R. Stokes, J. Exp. Biol. 203, 2713 (2000).
[9] N. Okamura and S. Ishiwata, J. Muscle Res. Cell Motil. 9, 111 (1988).

[10] T. Anazawa, K. Yasuda, and S. Ishiwata, Biophys. J. 61, 1099 (1992).

[11] K. Yasuda, Y. Shindo, and S. Ishiwata, Biophys. J. 70, 1823 (1996).

[12] H. Fujita and S. Ishiwata, Biophys. J. 75, 1439 (1998).

[13] C. Shingyoji, H. Higuchi, and T. Yanagida, Nature 393 (1998).

[14] K. A. P. Edman and N. A. Curtin, J. Physiol. 534, 553 (2001).

[15] F. Jülicher and J. Prost, Phys. Rev. Lett. 75, 2618 (1995).

[16] A. Vilfan, E. Frey, and F. Schwabl, Europhys. Lett. 45, 283 (1999).

[17] D. Riveline, et al., Eur. Biophys. J. 27, 403 (1998).

[18] S. A. Endow and H. Higuchi, Nature 406, 913 (2000).

[19] F. Jülicher and J. Prost, Phys. Rev. Lett. 78, 4510 (1997). 
[20] N. Thomas and R. A. Thornhill, J. Phys. D. 31, 253 (1998).

[21] F. Jülicher, C.R. Acad. Sci. (Paris) IV pp. 849-860 (2001).

[22] A. Pikovsky, M. Rosenblum, and J. Kurths, Synchronization, A Universal Concept in Nonlinear Sciences (Cambridge Univeristy Press, Cambridge, 2001).

[23] D. Golomb, D. Hansel, B. Shraiman, and H. Sompolinsky, Phys. Rev. A 45, 3516 (1992).
[24] V. Hakim and W.-J. Rappel, Phys. Rev. A 46, R7347 (1992).

[25] N. Nakagawa and Y. Kuramoto, Physica D 75, 74 (1994).

[26] Y. Kuramoto, Chemical Oscillations, Waves, and Turbulence (Springer, Berlin, 1984).

[27] D. A. Smith and D. G. Stephenson, J. Muscle Res. Cell Motil. 15, 369 (1994). 